\title{
Excessive Atrial Ectopic Activity Worsens Prognosis and Predicts the Type of Major Adverse Cardiac Events in Patients With Frequent Premature Ventricular Contractions
}

\author{
Leonor Parreira $^{\mathrm{a}, \mathrm{b}}$, Rita Marinheiro ${ }^{\mathrm{a}}$, Dinis Mesquita ${ }^{\mathrm{a}}$, Jose Farinha ${ }^{\mathrm{a}}$, Marta Fonseca ${ }^{\mathrm{a}}$, \\ Pedro Amador ${ }^{\mathrm{a}}$, Duarte Chambel ${ }^{\mathrm{a}}$, Artur Lopes ${ }^{\mathrm{a}}$, Rui Caria ${ }^{\mathrm{a}}$
}

\begin{abstract}
Background: The aim of the study was to evaluate the impact of premature atrial contractions (PACs) burden, and the presence of nonsustained ventricular tachycardia (NSVT) on prognosis and type of major adverse cardiovascular events in patients with frequent premature ventricular contractions (PVCs).

Methods: We retrospectively studied 285 consecutive patients with frequent PVCs defined as PVC count equal or higher than $1 \%$ of total beats assessed with 24-h Holter recording. Patients with atrial fibrillation (AF) were excluded. We evaluated the impact of PAC burden and the presence of NSVT on the primary end points of all-cause mortality, stroke and new-onset AF, and secondary end points; arrhythmic end point (arrhythmic death or hospitalizations for ventricular arrhythmias) or heart failure (HF)-related end point (death or hospitalizations due to $\mathrm{HF}$ ).
\end{abstract}

Results: The PAC number showed an adjusted hazard ratio (HR) (95\% confidence interval (CI), P value) of 1.077 (1.014 - 1.145, P $=0.017)$ for all-cause mortality, $1.250(1.080-1.447, \mathrm{P}=0.003)$ for stroke, $1.090(1.006-1.181, \mathrm{P}=0.036)$ for new-onset $\mathrm{AF}$ and $1.376(1.128-1.679, \mathrm{P}=0.002)$ for the HF end point. The presence of NSVT showed an adjusted HR (95\% CI) of 3.644 (1.147 - 11.57, P $=0.028$ ) for the arrhythmic end point.

Conclusions: In patients with frequent PVCs a high PAC count was independently associated with increased mortality, higher rate of AF, stroke and HF adverse events, but not with arrhythmic adverse events. The presence of NSVT was independently associated with increased arrhythmic adverse events, but not with overall mortality, AF, stroke or HF events.

Keywords: Atrial fibrillation; Excessive atrial ectopic activity; Heart failure; Premature atrial contractions; Premature ventricular contractions; Stroke

Manuscript submitted August 25, 2019, accepted September 6, 2019

${ }^{a}$ Cardiology Department, Centro Hospitalar de Setubal, Setubal, Portugal

${ }^{\mathrm{b}}$ Corresponding Author: Leonor Parreira, Av Dr Antonio Rodrigues Manito,

114 2900-064 Setubal, Portugal. Email: leonor.parreira@gmail.com

doi: https://doi.org/10.14740/cr935

\section{Introduction}

Premature ventricular contractions (PVCs) are a common finding during long-term monitoring [1]. Although PVCs can occur in healthy persons, they are more frequent in the presence of structural heart disease (SHD), sleep apnea [2], chronic obstructive pulmonary disease, and hyperthyroidism or stimulants [3]. Frequent PVCs in the presence of SHD are associated with a worse prognosis, especially in ischemic heart disease and in the presence of complex forms. Lown's grading of PVCs into six categories [4] was initially proposed as a prognostic classification in the acute myocardial infarction (MI) scenario. Currently this classification is no longer used as an indication for therapy, however some of the definitions are still used, like the severity based on the morphology or in the presence of runs of non-sustained ventricular tachycardia (NSVT). Recently the presence of frequent or severe forms of PVCs have been associated with increased mortality, heart failure (HF), atrial fibrillation and stroke, even in the absence of known SHD [5-8].

However, patients often have frequent premature atrial contractions (PAC), in addition to frequent PVCs as in the case of older populations, especially in the presence of cardiovascular risk factors [9]. PACs have been considered a benign incident for many years. Recent studies have demonstrated an association between PACs and atrial fibrillation (AF) and stroke [10-13]. In fact, frequent PACS have been considered an independent marker of stroke, adverse cardiovascular events and death, suggesting that the risk associated with frequent PACs is beyond the association with $\mathrm{AF}[10,12]$.

In previous studies that evaluated the role of frequent PVCs as predictors of outcomes like HF, new-onset AF and stroke $[7,8,14]$, none has assessed the role of concomitant frequent PACs. The aim of this study was to evaluate the impact of the number of PACs and the presence of NSVT on allcause mortality and on major adverse cardiovascular events (MACEs) in patients with frequent PVCs.

\section{Materials and Methods}

\section{Patients}

We evaluated 1,967 consecutive patients older than 18 years 
who underwent 24-h Holter monitoring in our center, between June 2006 and December 2010. We selected patients with frequent PVC, defined as PVCs representing more than $1 \%$ of total number of heart beats, because according to Dukes et al [5] this is the cut-off point that provides the best sensitivity/specificity relation for prediction of adverse outcomes $(n=530)$. Patients with AF, atrial flutter or any rhythm other than sinus during recording were excluded $(\mathrm{n}=168)$. Patients who were lost to follow-up $(\mathrm{n}=50)$ and patients with previous history of documented atrial AF or atrial flutter $(n=27)$ were further excluded. The final study population included 285 patients.

\section{Study design}

We retrospectively recorded the demographic data. From the medical records we collected the presence of diabetes, hypertension, hypercholesterolemia, antiarrhythmic and beta-blocker use, presence of SHD and its type. The $\mathrm{CHA}_{2} \mathrm{DS}_{2} \mathrm{VASc}$ risk was calculated according to the presence of congestive HF/left ventricular dysfunction (1 point); hypertension (1 point); age $\geq 75$ years ( 2 points); diabetes mellitus ( 1 point); history of stroke, transient ischemic attack (TIA) or thromboembolism (2 points); vascular disease (history of MI, vascular disease or aortic atherosclerosis) (1 point); age $65-74$ years (1 point) and female gender (1 point). We considered the presence of a high stroke risk if the $\mathrm{CHA}_{2} \mathrm{DS}_{2}$ VASc score was higher than 2 in men or 3 in women.

All transthoracic echocardiograms were retrospectively collected. Echocardiographic evaluation included M-mode measurements of the left atrium diameter (LAD), left ventricular end diastolic diameter (LVDD), left ventricular end systolic diameter (LVSD) and left ventricular fractional shortening (LVFS) obtained in the left parasternal view. Holter recording was performed with the use of three-channel tape recorders (GE SEER LIGHT ${ }^{\circledR}$ ). Recordings had to exceed $20 \mathrm{~h}$ and be of good quality to be analyzed and all of them were reviewed.

On Holter evaluation, maximal, minimal and mean heart rate, number of PACs/24 h (PACs/day), presence of supraventricular runs of more than five PACs, number of $\mathrm{PVCs} / 24 \mathrm{~h}$ (PVCs/day), morphology of the PVCs, polymorphic versus monomorphic, this variable was not available in 10 patients corresponding to $3.5 \%$ of the study population, presence of runs of more than five PVCs (NSVT) and presence of atrioventricular (AV) node disease were assessed.

Follow-up data were retrieved from the national patient registry and from medical records or discharge letters, and were validated by reviewing patients' files. Patients who failed to have recent clinical records were contacted by phone.

Death was ascertained by reviewing medical records, or from national patient registry.

HF death was defined as worsening HF, manifested as cardiogenic shock, pulmonary edema or increase in HF symptoms and drug therapy, or hospitalization due to decompensated HF prior to death. Arrhythmic death was defined as instantaneous death or death in patients who were resuscitated from a sudden cardiac arrest or occurred within $24 \mathrm{~h}$ of acute symptoms in the absence of pre-existing circulatory failure, and if unobserved, assumed to be acute and instantaneous.
Ischemic stroke was defined as a neurological deficit of sudden onset that persisted for more than $24 \mathrm{~h}$. It was confirmed by computerized axial tomography or magnetic resonance imaging of the brain. New-onset AF was defined as AF documented by a standard 12-lead electrocardiogram (ECG) or a new 24-h Holter monitoring. Hospitalization was defined as an overnight stay in a hospital ward. The cause of hospitalization was obtained from medical records, and cardiovascular hospitalization included the ones due to aggravated HF, ventricular arrhythmia, bradyarrhythmia or acute coronary syndromes. The primary end points determined were all-cause mortality, stroke and new-onset AF. Secondary end point was the type of MACE during follow-up: arrhythmic MACE; arrhythmic death or hospitalizations due to ventricular arrhythmias (VA), or HF-related MACE; death or hospitalizations due to HF.

\section{Ethics}

The Ethical Committee of the Centro Hospitalar de Setubal approved the study. The study is in compliance with the Helsinki Declaration. The informed consent was waived via the Ethical Committee.

\section{Statistical analysis}

All analyses were performed using SPSS statistical software, version 24.0 (SPSS, Inc, Chicago, Illinois). Data are expressed as median and interquartile range (IQR) for continuous variables due to lack of normal distribution, and as frequencies and percentages for categorical variables.

The relative risk for a given end point associated with the different variables studied was estimated by calculating the hazard ratio (HR) using a Cox regression hazards model. To adjust for potential confounders like age, gender, cardiovascular risk factors, $\mathrm{CHA}_{2} \mathrm{DS}_{2} \mathrm{VASc}$, presence of SHD, incident AF, LVFS, and LAD, multivariable Cox proportional-hazards model was used. The assumption of linearity for continuous covariates was tested and confirmed. We used a log (base 2) transformation of the PVC count and PAC count to meet model linearity assumptions. The proportional hazard assumption was tested for each of the Cox models based on Schoenfeld residuals for continuous variables and on "log-minus-log" plot for categorical variables.

Receiver operator characteristic curves (ROC) and areas under the curve (AUC) were obtained to determine the discriminative power of PAC burden as predictor of all-cause death $(\mathrm{AUC}=0.669, \mathrm{P}<0.0001 ; 95 \%$ confidence interval $(\mathrm{CI})$ (0.606 - 0.733). Optimal cut-point value for the number of PACs/day was obtained (Youden index) and patients were divided according to this value (Fig. 1). The optimum cut-point value for predicting mortality was 70 PACs on 24-h ECG monitoring (70 PACs/day), with sensitivity $61 \%$ and specificity of $65 \%, \mathrm{P}<0.0001$, which was considered excessive atrial ectopic activity (EAEA). Baseline characteristics in patients with and without EAEA and in patients with and without NSVT were compared using the Chi-square test for categorical vari- 


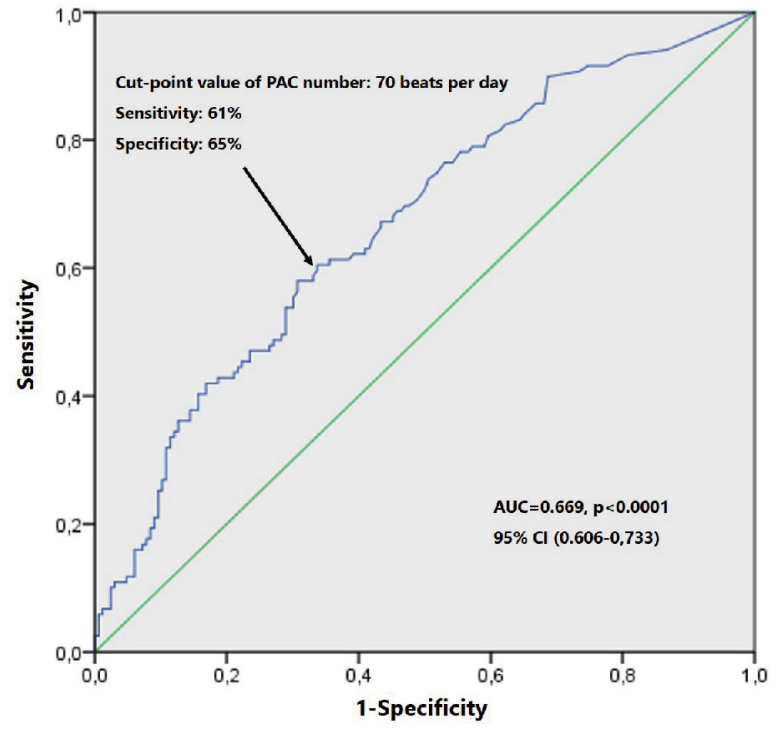

Figure 1. ROC curve survival analysis by PAC number. PAC: premature atrial contraction; ROC: receiver operator characteristic.

ables and the Mann-Whitney U test for continuous variables. Kaplan-Meier survival function was used to compare the event-free survival in the groups with and without EAEA and with and without NSVT. The Log- rank test (Mantel-Cox test) was used for comparison between groups.

\section{Results}

\section{Baseline characteristics}

The baseline characteristics of patients with and without EAEA are presented in Table 1. Patients with EAEA were generally older, with a higher incidence of hypertension, higher $\mathrm{CHA}_{2} \mathrm{DS}_{2}$ VASc score and were less frequently on beta-blockers. On the Holter recording patients with EAEA had lower maximal and mean heart rate, lower number of PVCs/day. The PVCs were more frequently polymorphic and finally, had more frequently runs of PACs. The echocardiographic evaluation showed larger LAD and larger LVDD. Patients with NSVT were more frequently male, had more SHD; the morphology of the PVCs was more frequently polymorphic and had larger left atrium and left ventricle (Table 2).

\section{Follow-up}

The median follow-up time was $8.4(5.1$ - 10) years, corresponding to 2,158 person-years. It was significantly shorter in the EAEA group, respectively, 7.6 (3.9 - 9.6) versus 8.7 (7.6 - 10.3) years, $\mathrm{P}<0.0001$, and in patients with NSVT, 7.8 (3.8 - 9.5) versus $8.6(5.5-10)$ years, $P=0.026$.

During the follow-up period, there were $119(42 \%)$ deaths, $10(4 \%)$ due to HF and eight (3\%) arrhythmic deaths, $19(7 \%)$ strokes. Sixty-four patients (25\%) developed AF. There were
$48(17 \%)$ cardiovascular (CV) hospitalizations, $14(5 \%)$ due to $\mathrm{HF}$ and five $(2 \%)$ due to ventricular arrhythmias. The numbers of events per 1,000 person-years according to the presence of EAEA or NSVT are displayed in Table 3.

\section{End points}

\section{Number of PACs and primary end points}

The number of PACs was associated with higher all-cause mortality, with an unadjusted estimated HR $(95 \% \mathrm{CI})$ of 1.131 (1.080 - 1.084), $\mathrm{P}<0.0001$ (Table 4). After adjustment to age, $\mathrm{CHA}_{2} \mathrm{DS}_{2} \mathrm{VASc}$ score, presence of SHD, beta-blocker therapy, new-onset AF, LAD and LVFS, the number of PACs was still associated with an increase in all-cause mortality with an estimated HR (95\% CI) of 1.077 (1.014 - 1.145), P = 0.017. The number of PACs was also associated with higher rates of stroke and new-onset AF unadjusted HR $(95 \% \mathrm{CI})$ of 1.321 (1.163 - 1.501), $\mathrm{P}<0.0001$ and 1.193 (1.117 - 1.275), $\mathrm{P}<$ 0.0001 respectively. After multivariable adjustment for confounding variables the number of PACS was still associated with a higher risk of stroke and new-onset AF, with an adjusted HR $(95 \% \mathrm{CI})$ of $1.250(1.080-1.447), \mathrm{P}=0.003$ for stroke and $1.090(1.006-1.181), \mathrm{P}=0.036$ for new-onset $\mathrm{AF}$.

\section{NSVT and primary end points}

The presence of NSVT episodes was associated with an increase in the risk of all-cause mortality with unadjusted HR (95\% CI) of 1.530 (1.001 - 2.337), $\mathrm{P}=0.049$ (Table 4). After adjustment to age, $\mathrm{CHA}_{2} \mathrm{DS}_{2} \mathrm{VASc}$ score, beta-blocker therapy, presence of SHD, new-onset AF, LAD and LVFS, the presence of episodes of NSVT was not associated with an increase in all-cause mortality.

\section{Number of PACs and secondary end points}

The number of PACs/day was associated with an increase in the risk of HF MACE with unadjusted HR $(95 \% \mathrm{CI})$ of 1.287 (1.119 - 1.482), $\mathrm{P}<0.0001$ (Table 4), and after adjustment to age, presence of SHD, beta-blocker therapy, new-onset AF and LVFS, the rate remained higher, with an adjusted HR $(95 \% \mathrm{CI})$ of 1.376 (1.128 - 1.679), $\mathrm{P}=0.002$. The number of PACs was not associated with the risk of arrhythmic MACE.

\section{NSVT and secondary end points}

The presence of NSVT was associated with the risk of HF and arrhythmic MACE with unadjusted HR $(95 \% \mathrm{CI})$ of 4.255 (1.542 - 11.74), $\mathrm{P}=0.049$ and 4.424 (1.484 - 13.19), $\mathrm{P}=0.008$ (Table 4). After adjustment for confounding variables, NSVT was associated with the rate of arrhythmic MACE with an adjusted HR (95\% CI) of 3.644 (1.147 - 11.57), $\mathrm{P}=0.028$ but not with HF MACE. 
Table 1. Baseline Characteristics in the Two Groups With and Without EAEA

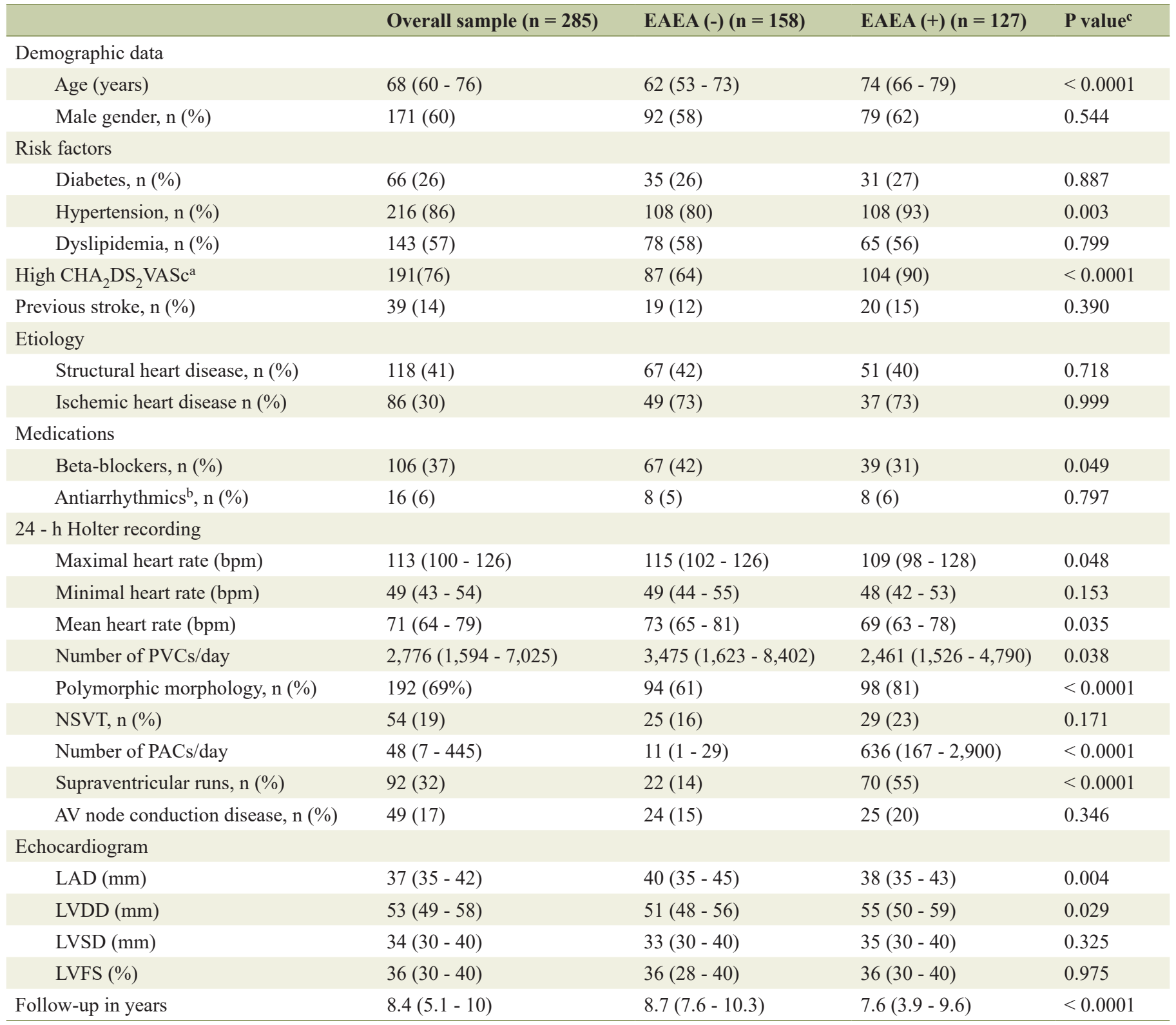

Values are presented as median (interquartile range) or $\mathrm{n}(\%)$. ${ }^{a}$ The $\mathrm{CHA}_{2} \mathrm{DS}_{2} \mathrm{VASc}$ score was calculated according to the presence of congestive heart failure/left ventricular dysfunction (1 point); hypertension ( 1 point); age $\geq 75$ years ( 2 points); diabetes mellitus ( 1 point); history of stroke, transitory ischemic attack or thromboembolism (2 points); vascular disease (history of MI, vascular disease or aortic atherosclerosis) (1 point); age 65 - 74 years (1 point) and female gender (1 point). ${ }^{b}$ Class I or class III antiarrhythmics. ${ }^{c} P$ values were calculated using Mann-Whitney $U$ test for continuous variables and the Chi-square test for categorical variables. EAEA: excessive atrial ectopic activity; NSVT: non-sustained ventricular tachycardia; PVC: premature ventricular contraction; PAC: premature atrial contraction; AV: atrioventricular; LAD: left atrium diameter; LVDD: left ventricular diastolic diameter; LVSD: left ventricular systolic diameter; LVFS: left ventricular fractional shortening.

\section{Predictors of risk}

\section{Presence of EAEA}

The numbers of events per 1,000 person-years in the groups with and without EAEA are shown in Table 3. Patients with EAEA had a worse prognosis, higher all-cause mortal- ity ( 83.2 versus 36.8 per 1,000 person-years), higher rate of stroke (18.8 versus 2.3 per 1,000 person-years), higher rate of new-onset AF (49.2 versus 16.9 per 1,000 person-years) and, higher rate of the HF MACE (16.4 versus 0.8 per 1,000 person-years). The EAEA was not associated with the rate of arrhythmic MACE.

They had a shorter overall survival (Fig. 2a), survival free from new-onset AF (Fig. 2c), survival free from stroke (Fig. 
Table 2. Baseline Characteristics in the Patients With and Without NSVT

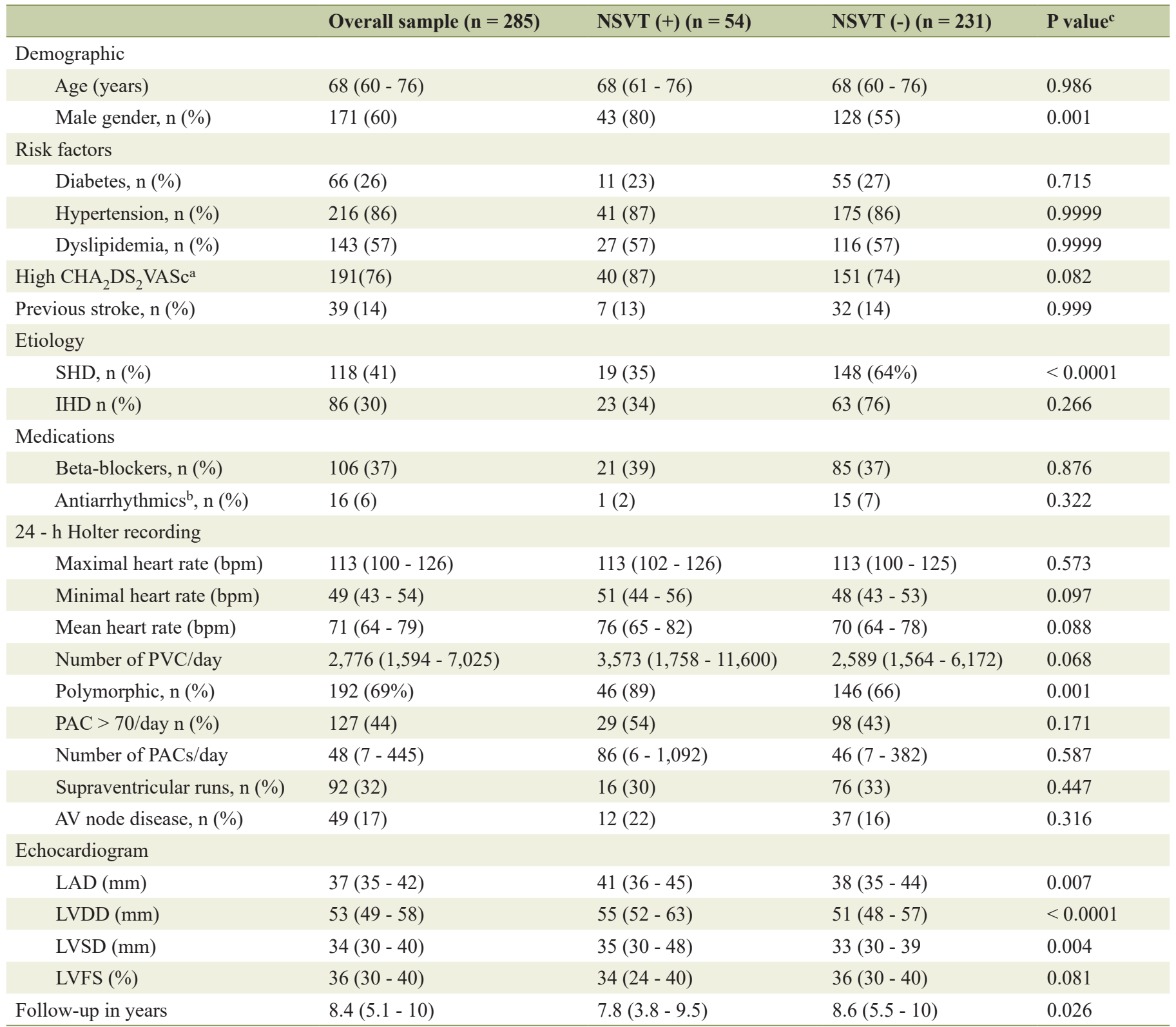

Values are presented as median (interquartile range) or $\mathrm{n}(\%)$. ${ }^{a} \mathrm{Th}_{\mathrm{CHA}} \mathrm{DS}_{2} \mathrm{VASc}$ score was calculated according to the presence of congestive heart failure/left ventricular dysfunction (1 point); hypertension (1 point); age $\geq 75$ years (2 points); diabetes mellitus (1 point); history of stroke, transitory ischemic attack or thromboembolism(2 points); vascular disease (history of MI, vascular disease or aortic atherosclerosis) (1 point); age 65 - 74 years (1 point) and female gender ( 1 point). ${ }^{\text {b}}$ Class I or class III antiarrhythmics. ${ }^{c} \mathrm{P}$ values were calculated using Mann-Whitney U test for continuous variables and the Chi-square test for categorical variables. EAEA: excessive atrial ectopic activity (> 70 PACs/day); SHD: structural heart disease; IHD: ischemic heart disease; NSVT: non-sustained ventricular tachycardia; PVC: premature ventricular contraction; PAC: premature atrial contraction; AV: atrioventricular; LAD: left atrium diameter; LVDD: left ventricular diastolic diameter; LVSD: left ventricular systolic diameter; LVFS: left ventricular fractional shortening.

2d), and survival free from HF death or HF hospitalizations (Fig. 3a).

\section{Presence of NSVT}

The numbers of events per 1,000 person-years in the groups with and without NSVT are shown in Table 3. Patients with NSVT had higher all-cause mortality ( 78 versus 50.5 per 1,000 person-years), higher rate of the HF MACE (19.5 versus 4.4 per 1,000 person-years) and higher rate of the arrhythmic MACE of arrhythmic death or VA hospitalizations (16.8 versus 5.5 per 1,000 person-years). The presence of NSVT was not significantly associated with a higher rate of stroke or new-onset AF. 
Table 3. Events per 1,000 Person-Years According to the Presence of EAEA or NSVT

\begin{tabular}{|c|c|c|c|c|}
\hline & Overall sample & EAEA (-) & EAEA (+) & Pyalue \\
\hline & 2,158 person-years & 1,305 person-years & 853 person-years & \\
\hline All-cause death & 55 & 36.8 & 83.2 & $<0.0001$ \\
\hline New-onset AF & 29.7 & 16.9 & 49.2 & $<0.0001$ \\
\hline HF death or HF hospitalizations & 7 & 0.8 & 16.4 & $<0.0001$ \\
\hline & Overall sample & NSVT (-) & NSVT (+) & Pyoluoa \\
\hline & 2,158 person-years & 1,800 person-years & 358 person-years & Pvalue \\
\hline All-cause death & 55 & 50.5 & 78 & 0.047 \\
\hline Stroke & 8.8 & 7.2 & 16.6 & 0.670 \\
\hline
\end{tabular}

Values are presented in number of events per 1,000 person-years. AF; atrial fibrillation; HF: heart failure; VA: ventricular arrhythmia; EAEA: excessive atrial ectopic activity; EAEA excessive atrial ectopic activity; NSVT: non-sustained VT episodes; NSVT: non-sustained VT episodes. aP values were calculated using the Log-rank test.

Patients with NSVT had a shorter overall survival (Fig. 2b), shorter survival free from HF death or HF hospitalizations (Fig. 3b) and shorter survival free from arrhythmic death or VA hospitalizations (Fig. 3d).

\section{Discussion}

Our study population was old and almost half of them had structural heart disease which may explain the high mortality and cardiovascular event rates. One of the inclusion criteria to enter the study was having a minimum of $1 \%$ of ventricular beats that were ectopic. So according to previous studies, this is a population with a higher mortality and higher risk of cardiovascular adverse events $[5,6,15]$. We observed that the presence of NSVT was independently associated with increased all-cause mortality, and with arrhythmic death or VA hospitalizations. Patients with NSVT were older and sicker, yet after

Table 4. Cox Regression Models Showing the Hazard Ratio of Number of PACs, and Presence of NSVT in Relation to the Primary and Secondary End Points

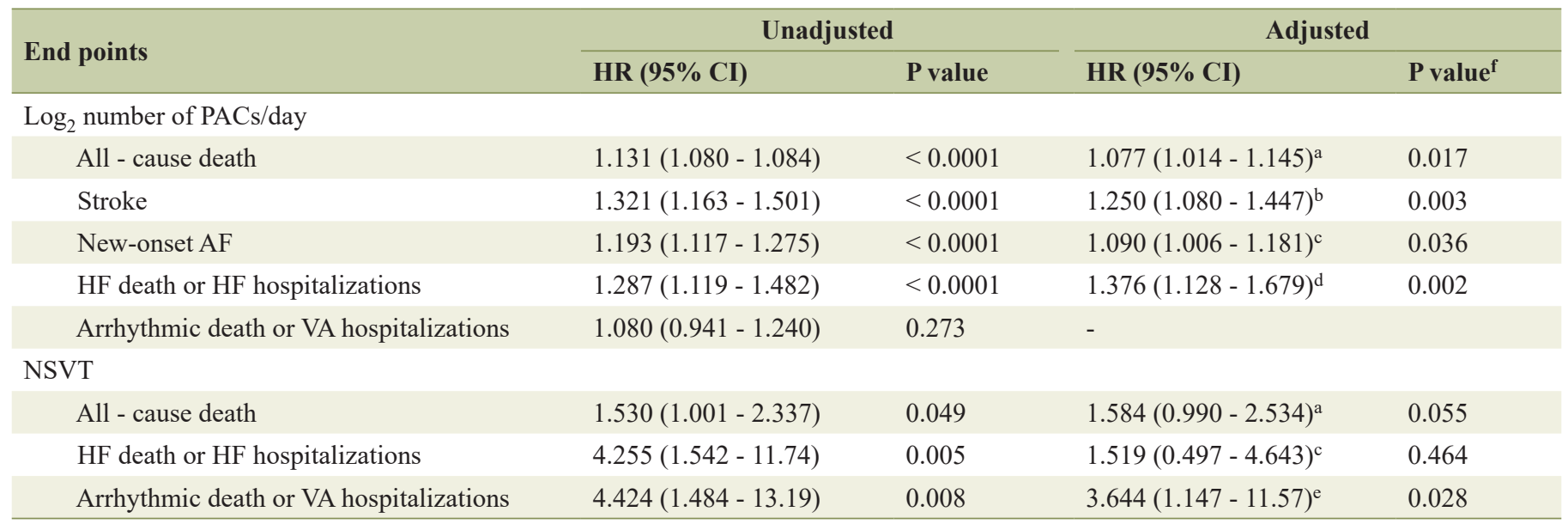

aHR adjusted to age, $\mathrm{CHA}_{2} \mathrm{DS}_{2} \mathrm{VASc}$ score, presence of SHD, beta-blocker therapy,new-onset AF, LAD and LVFS. bHR adjusted to age, CHA ${ }_{2} \mathrm{DS}$ ${ }_{2} \mathrm{VASc}$ score, previous stroke, LAD and new-onset AF. cHR adjusted to age, hypertension, diabetes, beta-blocker therapy, presence of SHD and LAD. dHR adjusted to the presence of age, SHD, beta-blocker therapy, new-onset AF and LVFS. eHR adjusted to the presence of age, beta-blocker therapy, structural heart disease and LVFS. ${ }^{\mathrm{f}} \mathrm{P}$ values were calculated using the Cox proportional-hazards model. PAC: premature atrial contraction; AF: atrial fibrillation; HF: heart failure; VA: ventricular arrhythmia; NSVT: non-sustained ventricular tachycardia; LAD: left atrium diameter; LVFS: left ventricular fractional shortening; SHD: structural heart disease. 

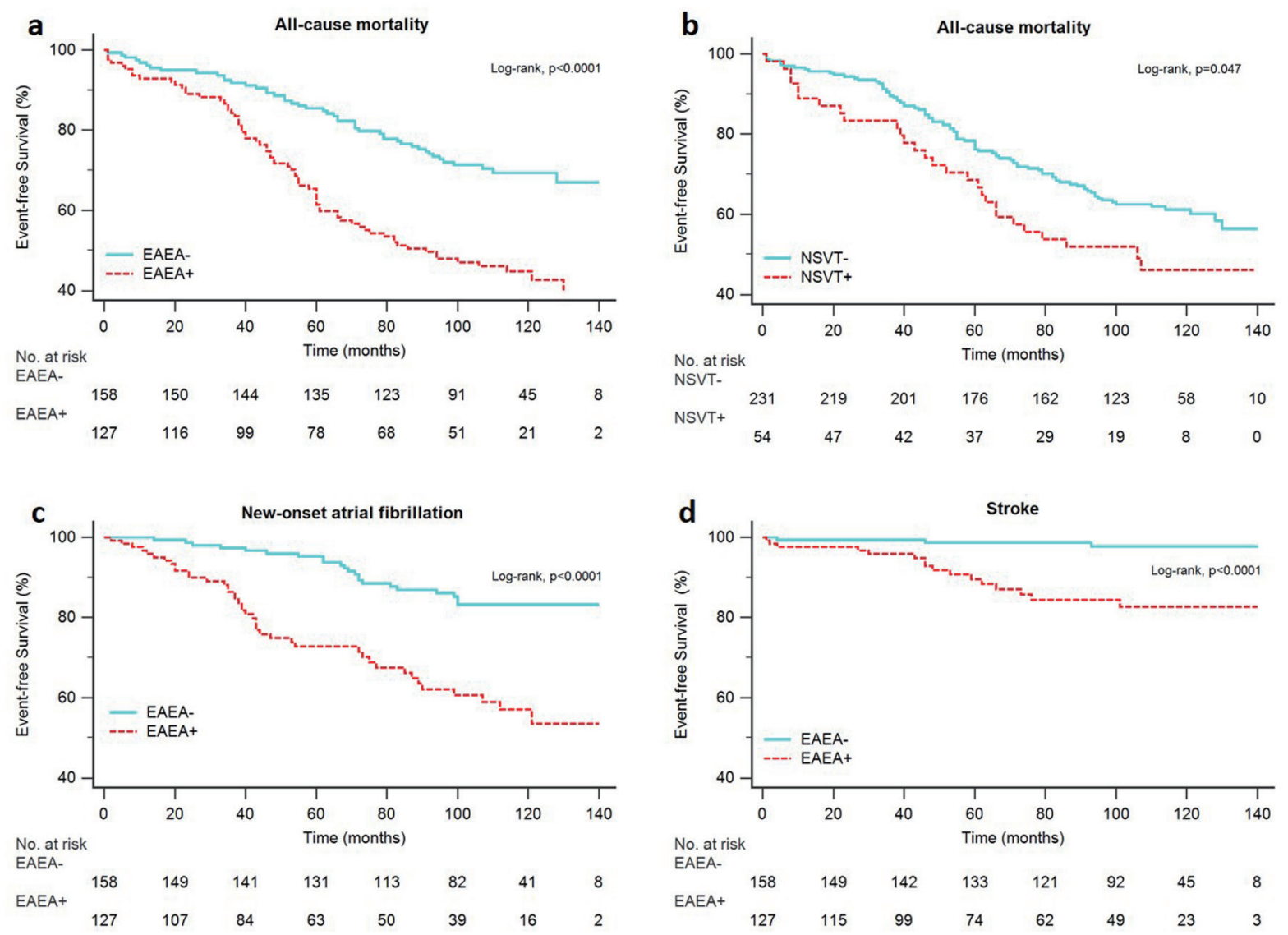

Figure 2. Kaplan-Meier estimates for the primary end points. Overall survival in patients with and without EAEA (a), overall survival in patients with and without NSVT (b), AF free survival in patients with and EAEA (c), stroke free survival in patients with and without EAEA (d). EAEA: excessive atrial ectopic activity; NSVT: non-sustained ventricular tachycardia.

adjustment to other covariables the presence of NSVT was still associated with arrhythmic death or VA hospitalizations.

In a recent work, Lin et al [8] followed 3,767 patients with apparently normal hearts for $10 \pm 1$ year and found that patients with NSVT had an increased risk of mortality, cardiovascular hospitalization, ischemic stroke, and new-onset HF.

In our group of patients, we also found a higher rate of HF death or HF hospitalizations in patients with NSVT. However, this effect is lost when adjusted to other risk variables.

Dukes et al [5] found an increase in mortality in patients with frequent PVCs that according to the authors appeared to be partly explained by incident HF. Left ventricular dysfunction induced by frequent PVCs has been described previously [16] although the underlying mechanism is still a matter of debate. Studies with catheter ablation have demonstrated left ventricular dysfunction in patients with a high PVC burden which is reversible after successful catheter ablation [17].

However, in most of these studies left ventricular dysfunction has been associated with a greater PVC burden, higher than $10 \%$ and usually higher than $20 \%$ of total beats $[16,17]$.

Therefore, in the presence of a lower PVC burden as is the case of our study, with PVCs representing a median of 2.7\% of all heart beats (IQR: 1.65-6.8 \%) or that of Dukes et al with PVCs representing a median of $0.011 \%$ of all heart beats (IQR: $0.002-0.123 \%$ ) [5], or in the study by Lin et al [8] with a mean PVC burden of $176 \pm 423$ beats per day, the possibility that the increase in HF rate might be due to PVCs is questionable. Also intriguing is the association between frequent PVCs and stroke described by Lin et al $[7,8]$ and previously reported by Agarwal et al [14]. In the latter, the authors found a stronger association between frequent PVCs and embolic stroke than thrombotic stroke. Therefore, the authors hypothesized that the reason for that might have been an increased risk of AF in those patients, and even suggested that patients with high PVC burden could be monitored for AF.

In our study the presence of NSVT was not associated with stroke or new-onset AF. On the other hand, the number of PACs was associated with an increased risk of stroke and new-onset AF, both before and after multivariable adjustment.

In all those previous studies that reported increased risk of HF, stroke and AF due to high PVC burden, the authors did not address the presence of frequent PACs in association with frequent or complex PVCs. To the best of our knowledge we present for the first time a study that demonstrates the role of PAC burden as a marker of prognosis in patients with frequent PVCs and is capable of explaining the high rate of HF events, 
a

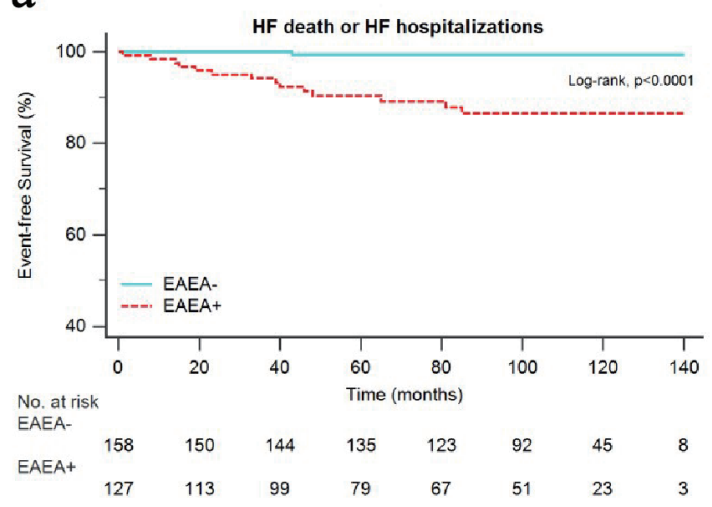

C

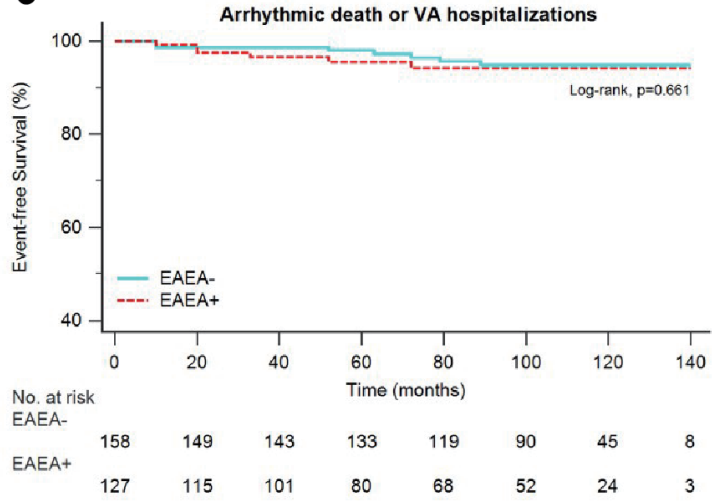

b
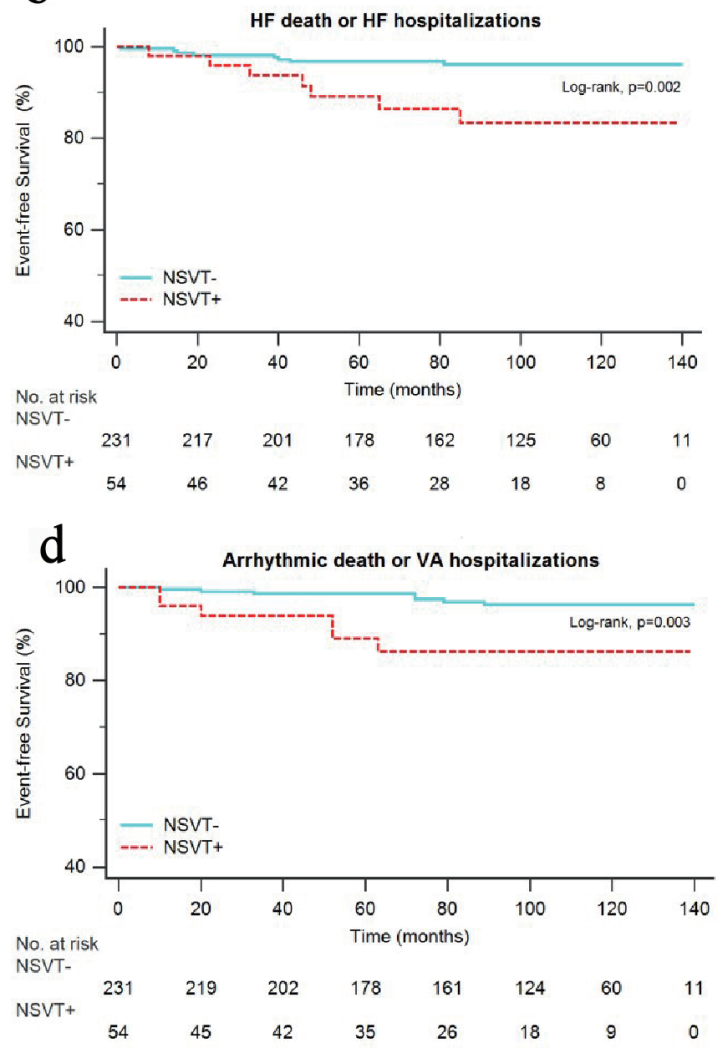

Figure 3. Kaplan-Meier estimates for the secondary end points. Survival free from HF death or HF hospitalizations in patients with and without EAEA (a), survival free from HF death or HF hospitalizations in patients with and without NSVT (b), survival free from arrhythmic death or VA hospitalizations in patients with and without EAEA (c), survival free from arrhythmic death or VA hospitalizations in patients with and without NSVT (d). EAEA: excessive atrial ectopic activity; HF: heart failure; NSVT: non-sustained ventricular tachycardia; VA: ventricular arrhythmia.

stroke or AF occurrence in patients with PVCs.

Patients with EAEA were older, had more hypertension and larger left atrium dimension. Old age is a recognized risk factor for development of AF and for PACs [9]. Several studies have shown that PACs and subclinical atrial arrhythmias are independent predictors of AF and stroke [10, 11, 13]. Some authors proposed that frequent PACs could be a marker of a higher prevalence of cardiovascular risk factors such as hypertension, diabetes, dyslipidemia and that could explain the higher incidence of stroke, independently of the development of AF $[11,12]$. In our study population, patients with frequent PACs were older, had more frequently hypertension, and had a higher CHA2DS2VASc score. However, the number of PACs was independently associated with the risk of stroke. One explanation for this fact might be that EAEA may lead to the dilatation of left atrium and stasis in the left atrial appendage, fibrosis and endothelial dysfunction resulting in a hypercoagulable state similar to that seen in AF [18]. Likewise, subclinical atrial arrhythmias like device-detected atrial high rate episodes have been related to an increased risk of AF and stroke [19, 20].

The presence of frequent PACs on the other hand is not associated with arrhythmic events as opposed to the presence of NSVT that was associated with an adjusted 3.6-fold higher rate of arrhythmic death or VA hospitalizations.

These results, if replicated in other future studies with a higher number of patients may have potential clinical implications and shed some light into the mechanisms of high rates of $\mathrm{HF}$, stroke and $\mathrm{AF}$ in patients with frequent PVCs.

\section{Limitations}

There were some limitations to our study. First of all, it was an observational and retrospective study and the high cardiovascular adverse event rate may result from a type of reference bias. The cut-point value of PVCs equal or higher than $1 \%$ of total beats may have selected a very high-risk population, although by using multivariable regression, we aimed at overcoming this limitation. We cannot exclude the presence of other risk factors that were not assessed like smoking habits, sleep apnea or chronic obstructive pulmonary disease. Further prospective studies with a higher number of patients are needed. 


\section{Conclusions}

In conclusion, in this group of patients with frequent PVCs, the presence of EAEA was independently associated with increased mortality, higher rate of incident AF, stroke, HF death or HF hospitalization but not with arrhythmic death or VA hospitalizations. Patients with NSVT had also a worse prognosis, and were more likely to have arrhythmic events. However, the presence of NSVT was not independently associated with increased all-cause mortality, a higher risk of HF events, stroke or new-onset AF.

\section{Acknowledgments}

None to declare.

\section{Financial Disclosure}

None to declare.

\section{Informed Consent}

The informed consent was waived via the Ethical Committee.

\section{Conflict of Interest}

None to declare.

\section{Author Contributions}

Leonor Parreira contributed to conception and design of the study, analysis and interpretation of data drafting and revising the article. Rita Marinheiro was involved in acquisition of data and revising the article. Dinis Mesquita was involved in acquisition of data and revising the article. Jose Farinha was involved in acquisition of data. Marta Fonseca was involved in acquisition of data. Pedro Amador was involved in acquisition of data. Duarte Chambel was involved in acquisition of data. Artur Lopes was involved in acquisition of data. Rui Caria, MD was involved in revising the article.

\section{References}

1. Arnar DO, Mairesse GH, Boriani G, Calkins H, Chin A, Coats A, Deharo JC, et al. Management of asymptomatic arrhythmias: a European Heart Rhythm Association (EHRA) consensus document, endorsed by the Heart Failure Association (HFA), Heart Rhythm Society (HRS), Asia Pacific Heart Rhythm Society (APHRS), Cardiac Arrhythmia Society of Southern Africa (CASSA), and Latin America Heart Rhythm Society (LAHRS). EP Europace. 2019:euz046.
2. Marinheiro R, Parreira L, Amador P, Mesquita D, Farinha J, Fonseca M, Duarte T, et al. Ventricular arrhythmias in patients with obstructive sleep apnea. Curr Cardiol Rev. 2019;15(1):64-74.

3. Almeneessier AS, Alasousi N, Sharif MM, Pandi-Perumal SR, Hersi AS, BaHammam AS. Prevalence and predictors of arrhythmia in patients with obstructive sleep apnea. Sleep Sci. 2017;10(4):142-146.

4. Lown B, Wolf M. Approaches to sudden death from coronary heart disease. Circulation. 1971;44(1):130-142.

5. Dukes JW, Dewland TA, Vittinghoff E, Mandyam MC, Heckbert SR, Siscovick DS, Stein PK, et al. Ventricular ectopy as a predictor of heart failure and death. J Am Coll Cardiol. 2015;66(2):101-109.

6. Agarwal V, Vittinghoff E, Whitman IR, Dewland TA, Dukes JW, Marcus GM. Relation between ventricular premature complexes and incident heart failure. Am J Cardiol. 2017;119(8):1238-1242.

7. Lin CY, Chang SL, Lin YJ, Lo LW, Chung FP, Chen YY, Chao TF, et al. Long-term outcome of multiform premature ventricular complexes in structurally normal heart. Int J Cardiol. 2015;180:80-85.

8. Lin CY, Chang SL, Chung FP, Chen YY, Lin YJ, Lo LW, $\mathrm{Hu}$ YF, et al. Long-term outcome of non-sustained ventricular tachycardia in structurally normal hearts. PLoS One. 2016;11(8):e0160181.

9. Kerola T, Dewland TA, Vittinghoff E, Heckbert SR, Stein PK, Marcus GM. Predictors of atrial ectopy and their relationship to atrial fibrillation risk. Europace. 2019;21(6):864-870.

10. Marinheiro R, Parreira L, Amador P, Sa C, Duarte T, Caria $\mathrm{R}$. Excessive atrial ectopic activity as an independent risk factor for ischemic stroke. Int J Cardiol. 2017;249:226230.

11. Binici Z, Intzilakis T, Nielsen OW, Kober L, Sajadieh A. Excessive supraventricular ectopic activity and increased risk of atrial fibrillation and stroke. Circulation. 2010;121(17):1904-1911.

12. Larsen BS, Kumarathurai P, Falkenberg J, Nielsen OW, Sajadieh A. Excessive atrial ectopy and short atrial runs increase the risk of stroke beyond incident atrial fibrillation. J Am Coll Cardiol. 2015;66(3):232-241.

13. Chong BH, Pong V, Lam KF, Liu S, Zuo ML, Lau YF, Lau CP, et al. Frequent premature atrial complexes predict new occurrence of atrial fibrillation and adverse cardiovascular events. Europace. 2012;14(7):942-947.

14. Agarwal SK, Heiss G, Rautaharju PM, Shahar E, Massing MW, Simpson RJ, Jr. Premature ventricular complexes and the risk of incident stroke: the Atherosclerosis Risk In Communities (ARIC) Study. Stroke. 2010;41(4):588593.

15. Engel G, Cho S, Ghayoumi A, Yamazaki T, Chun S, Fearon WF, Froelicher VF. Prognostic significance of PVCs and resting heart rate. Ann Noninvasive Electrocardiol. 2007;12(2):121-129.

16. Baman TS, Lange DC, Ilg KJ, Gupta SK, Liu TY, Alguire $\mathrm{C}$, Armstrong W, et al. Relationship between burden of premature ventricular complexes and left ventricular function. Heart Rhythm. 2010;7(7):865-869. 
17. Bogun F, Crawford T, Reich S, Koelling TM, Armstrong W, Good E, Jongnarangsin K, et al. Radiofrequency ablation of frequent, idiopathic premature ventricular complexes: comparison with a control group without intervention. Heart Rhythm. 2007;4(7):863-867.

18. Watson T, Shantsila E, Lip GY. Mechanisms of thrombogenesis in atrial fibrillation: Virchow's triad revisited. Lancet. 2009;373(9658):155-166.
19. Healey JS, Connolly SJ, Gold MR, Israel CW, Van Gelder IC, Capucci A, Lau CP, et al. Subclinical atrial fibrillation and the risk of stroke. N Engl J Med. 2012;366(2):120129.

20. Marinheiro R, Parreira L, Amador P, Lopes C, Fernandes A, Mesquita D, Farinha J, et al. Clinical impact of oral anticoagulation in patients with atrial high-rate episodes. J Stroke Cerebrovasc Dis. 2019;28(4):971-979. 